\title{
Electron pre-acceleration through stochastic shock drift acceleration at intracluster shocks
}

\author{
Jacek Niemiec, ${ }^{a, *}$ Oleh Kobzar, ${ }^{b, c}$ Karol Fulat, ${ }^{d}$ Martin Pohl, $, e, f$ Takanobu Amano, ${ }^{g}$ \\ Masahiro Hoshino, ${ }^{g}$ Shuichi Matsukiyo ${ }^{h}$ and Yosuke Matsumoto ${ }^{i}$
}

${ }^{a}$ Institute of Nuclear Physics Polish Academy of Sciences, PL-31342 Krakow, Poland

${ }^{b}$ Faculty of Materials Engineering and Physics, Cracow University of Technology, PL-30084 Kraków, Poland

${ }^{c}$ Astronomical Observatory of the Jagiellonian University, PL-30244 Kraków, Poland

${ }^{d}$ AGH University of Science and Technology, Faculty of Physics and Applied Computer Science, PL-30059 Kraków, Poland

e Institute of Physics and Astronomy, University of Potsdam, DE-14476 Potsdam, Germany

${ }^{f}$ DESY, DE-15738 Zeuthen, Germany

${ }^{g}$ Department of Earth and Planetary Science, the University of Tokyo, Tokyo 113-0033, Japan

${ }^{h}$ Faculty of Engineering Sciences, Kyushu University, Fukuoka, 816-8580, Japan

${ }^{i}$ Department of Physics, Chiba University, Chiba 263-8522, Japan

E-mail: jacek.niemiec@ifj.edu.pl

Radio and X-ray observations of radio relics indicate acceleration of relativistic electrons at merger shocks in galaxy clusters. These large-scale shocks can also be sites of ultra-high-energy cosmic ray production. It is assumed that diffusive shock acceleration (DSA) produces synchrotronradiating electrons but the process of electron pre-acceleration from thermal to supra-thermal energies is poorly known. Using large-scale fully-kinetic two-dimensional particle-in-cell (PIC) simulations of a quasi-perpendicular subluminal shock with low sonic Mach number $\left(M_{S}=3\right)$ and propagating in hot intracluster medium with plasma beta $\beta=5$ we have recently demonstrated that the main electron pre-acceleration mechanism is stochastic shock-drift acceleration (SSDA). In this process electrons are confined at the shock by pitch-angle scattering off turbulence and gain energy while drifting along the motional electric field. We showed that multi-scale magnetic turbulence, including ion-scale shock rippling modes, is essential for electron energization. This turbulence is driven by effective ion and electron temperature anisotropies in the entire shock transition. Wide-energy non-thermal electron distributions are formed both upstream and downstream of the shock and the maximum energy of the electrons is sufficient for their injection into DSA. Here we report on our new PIC simulation studies of SSDA process in a range of plasma beta $(\beta=5-30)$ and subluminal shock obliquity angles. We show that SSDA persists in facilitating the electron injection in rippled shocks. We also present how the SSDA efficiency vary with intracluster medium and shock parameters.

$3^{\text {th }}$ International Cosmic Ray Conference (ICRC 2021)

July 12th-23rd, 2021

Online - Berlin, Germany

\footnotetext{
*Presenter
} 


\section{Introduction}

Observations of radio relics in radio and X-rays indicate acceleration of relativistic electrons at merger shocks in galaxy clusters [1, 14]. These large-scale shocks can also be sites of ultra-highenergy cosmic ray (UHECR) production. The most energetic merger shocks have low sonic Mach numbers, $M_{\mathrm{s}} \lesssim 5$, and propagate in hot intracluster medium (ICM), in which plasma beta - a ratio of thermal to magnetic pressure - is high $(\beta \gg 1)$. The plausible particle acceleration process is Diffusive Shock Acceleration (DSA). One of the most critical ingredients of this process is the mechanism of electron injection which is poorly known for ICM conditions.

A widely discussed electron injection mechanism at ICM shocks is Shock Drift Acceleration (SDA) [10]. It has been recently recognized that electrons reflected from the shock in the SDA process can generate their own turbulence in the shock upstream $[3,4,6,11]$. These waves are driven by the electron firehose instability (EFI) and can scatter electrons back towards the shock enabling another interaction with the shock. It was shown that this multiple-cycle SDA process can lead to the formation of upstream supra-thermal electron spectra and works efficiently at highbeta shocks, though maximum energies are smaller than the estimated injection threshold to DSA. Using large-scale particle-in-cell (PIC) simulations of a quasi-perpendicular subluminal shock with $M_{s}=3$ and $\beta=5$ we have recently demonstrated that the main electron pre-acceleration mechanism to the injection energy is stochastic SDA (SSDA) [7, 9]. Here we report on our new PIC simulation studies in a range of plasma beta $(\beta=5-30)$ and subluminal shock obliquity angles.

\section{Simulation setup}

We use a 2D3V version of MPI-parallelized and optimized relativistic electromagnetic PIC code TRISTAN $[2,13]$. An electron-ion plasma beam is injected at the right side of the computational box in the $x-y$ plane and flows with a bulk velocity $v_{0}=0.1 c$ in the $-x$-direction. The collision of the inflowing plasma with the beam reflected off the conductive wall at the left boundary spawns a shock propagating in the $+x$-direction with velocity $v_{\mathrm{sh}}$. The injected plasma carries a large-scale magnetic field, $\boldsymbol{B}_{0}$, that lies in the simulation plane at the angle $\theta_{\mathrm{Bn}}$ with respect to the shock normal. With the magnetic field a motional electric field $\boldsymbol{E}=-\left[\boldsymbol{v}_{0} \times \boldsymbol{B}_{0}\right]$ is initialized and has only out-of-plane component, $E_{0 z}$.

Table 1 lists parameters of simulation runs discussed in this paper. They are chosen to represent physical conditions at weak supercritical ICM shocks [6], and so we assume the sonic Mach number of the shocks measured in the upstream rest frame, $M_{\mathrm{s}} \equiv v_{\mathrm{sh}}^{\mathrm{up}} / c_{\mathrm{s}}=3$. Here, $c_{\mathrm{s}}=\sqrt{2 \Gamma k_{\mathrm{B}} T_{\mathrm{i}} / m_{\mathrm{i}}}$ is the sound speed, and $\Gamma=5 / 3$ is the adiabatic index. Electrons are initially in thermal equilibrium with ions, $T_{\mathrm{e}}=T_{\mathrm{i}}=T_{0} \approx 5 \cdot 10^{8} \mathrm{~K}=43 \mathrm{keV} / k_{\mathrm{B}}$. We also consider quasi-perpendicular and subluminal shocks. The critical superluminality angle is $\theta_{\mathrm{Bn}, \mathrm{cr}}=\cos ^{-1}\left(v_{\mathrm{sh}}^{\mathrm{up}} / c\right) \approx 81.4^{\circ}$. Following [9], we assume that the thermal speed of upstream electrons $v_{\text {th,e }} \lesssim v_{\mathrm{t}}$, where $v_{\mathrm{t}}=v_{\mathrm{sh}}^{\text {up }} / \cos \theta_{\mathrm{Bn}}$ is de Hoffman-Teller velocity [3]. In such conditions few electrons are reflected which results in inefficient EFI wave generation. The simulation discussed in [9] (run A) uses $\theta_{\mathrm{Bn}}=75^{\circ}$, for which $v_{\mathrm{t}} \approx 1.5 v_{\text {th,e }}$. Consequently, low-amplitude EFI waves are observed in the laminar shock phase. However, EFI modes are significantly amplified upon the emergence of the shock ripples. They change the local obliquity angle along the shock surface below the limiting angle 


\begin{tabular}{c|c|c|c|c|c|c}
\hline \hline Run & $\beta$ & $\theta_{\mathrm{Bn}}\left[^{\circ}\right]$ & $M_{A}$ & $\lambda_{s i}[\Delta]$ & $L_{y}\left[\lambda_{s i}\right]$ & $t_{\max }\left[\Omega_{i}^{-1}\right]$ \\
\hline $\mathrm{A}$ & 5 & 75 & 6.1 & 150 & 32 & 78 \\
$\mathrm{~B}$ & 5 & 78 & 6.1 & 100 & 24 & 50 \\
$\mathrm{C} 1$ & 5 & 75 & 6.1 & 100 & 12 & 50 \\
$\mathrm{C} 2$ & 10 & 75 & 8.7 & 100 & 12 & 50 \\
$\mathrm{C} 3$ & 20 & 75 & 12.3 & 100 & 12 & 50 \\
$\mathrm{C} 4$ & 30 & 75 & 15.0 & 100 & 12 & 50 \\
$\mathrm{D}$ & 20 & 75 & 12.3 & 100 & 36 & 50 \\
\hline
\end{tabular}

Table 1: Parameters of simulation runs: plasma beta, $\beta$, the inclination angle of the upstream in-plane magnetic field to the shock normal, $\theta_{\mathrm{Bn}}$, the Alfvénic Mach number, $M_{\mathrm{A}}$, the ion skin depth, $\lambda_{\mathrm{si}}$, in units of the cell size, $\Delta$, the transverse size of the computational box, $L_{y}$, in units of $\lambda_{\mathrm{si}}$, and the maximum simulation time, $t_{\text {max }}$.

of $\theta_{\text {limit }}=\arccos \left(M_{\mathrm{s}} \sqrt{\Gamma m_{\mathrm{e}} / m_{\mathrm{i}}}\right) \simeq 67^{\circ}$, enabling localized efficient electron reflection and EFI driving. In run $\mathrm{B}$ we set the magnetic field obliquity angle to $\theta_{\mathrm{Bn}}=78^{\circ}$. In this case $v_{\mathrm{t}} \approx 1.9 v_{\text {th,e }}$ and EFI waves are not excited even in the rippled shock (see below).

In runs $\mathrm{A}$ and $\mathrm{B}$, the total plasma beta, $\beta \equiv p_{\text {th }} / p_{\mathrm{m}}=2 \mu_{0}\left(N_{\mathrm{e}}+N_{\mathrm{i}}\right) k_{\mathrm{B}} T_{0} / B_{0}^{2}$, is equally carried by electrons and ions, $\beta_{\mathrm{e}}=\beta_{\mathrm{i}}$, and set to $\beta=5$. Here, $\mu_{0}$ is the vacuum permeability, and $N_{\mathrm{i}}$ and $N_{\mathrm{e}}$ are the upstream ion and electron number densities. We also study the SSDA process in conditions of higher plasma beta, $\beta=5-30$, which is more realistic for ICM shocks. Runs C1-C4 explore shocks with $\beta=5,10,20$ and 30 using relatively narrow simulation boxes with the transverse size of $L_{y}=12 \lambda_{\mathrm{si}}$, where $\lambda_{\mathrm{si}}$ is the ion skin depth. These runs do not fully resolve the ripple modes at the shock surface, but enable us to reliably trace the shock structure and features of electron acceleration at small scales and shorter time-scales. This has been verified through comparison of run $\mathrm{C} 1$ with run A, that differ only in $L_{y}$. For further investigations of a case with high plasma beta we perform run $\mathrm{D}$ with $\beta=20$ and large-size computational grid, $L_{y}=36 \lambda_{s i}$, fully capturing the ion-scale fluctuations.

To obtain different $\beta$ in runs $\mathrm{C} 1-\mathrm{C} 4$ and $\mathrm{D}$ we fix the plasma temperature and change the magnetic field strength. Resulting shock Alfveń Mach numbers, $M_{\mathrm{A}}=v_{\mathrm{sh}}^{\text {up }} / v_{\mathrm{A}}$, where $v_{\mathrm{A}}=$ $B_{0} / \sqrt{\mu_{0}\left(N_{e} m_{e}+N_{i} m_{i}\right)}$ is the the Alfvén velocity, are listed in Table 1 . We apply a reduced ionto-electron mass ratio $m_{\mathrm{i}} / m_{\mathrm{e}}=100$. For the electron skin depth, $\lambda_{\text {se }} \equiv c / \omega_{\text {pe }}$, we use $\lambda_{\text {se }}=15 \Delta$ in run $\mathrm{A}$ and $\lambda_{\mathrm{se}}=10 \Delta$ in other runs, where $c$ is the speed of light, $\omega_{\mathrm{pe}}=\sqrt{e^{2} N_{e} / \epsilon_{0} m_{\mathrm{e}}}$ is the electron plasma frequency, $e$ the electron charge, $\epsilon_{0}$ the vacuum permittivity, and $\Delta$ the cell size. We use 20 particles per cell per species. The ion skin depth, $\lambda_{\mathrm{si}}=\sqrt{m_{\mathrm{i}} / m_{\mathrm{e}}} \lambda_{\mathrm{se}}$, is our unit of length. Time is in units of inverse upstream ion gyrofrequency, $\Omega_{\mathrm{i}}=e B_{0} / m_{\mathrm{i}}$.

\section{Results}

Figure 1 shows the shock structure for run A (left panel), run B (middle panel) and run D (right panel) at time $\Omega_{\mathrm{i}} t=36$. At this stage the shock ripples are already well developed, and one can see the overshoot-undershoot structures characteristic of a quasi-perpendicular shock. In particular, in run A the first overshoot is located at $x \approx 80 \lambda_{s i}$, the undershoot at $x \approx 73 \lambda_{s i}$, and the second overshoot at $x \approx 70 \lambda_{s i}$. One can note the presence of multi-scale turbulence, whose features are similar in all cases studied. This is due to ion and electron temperature anisotropies in the shock transition, which provide comparable conditions for excitation of various plasma instabilities that generate waves of different wavelengths. 

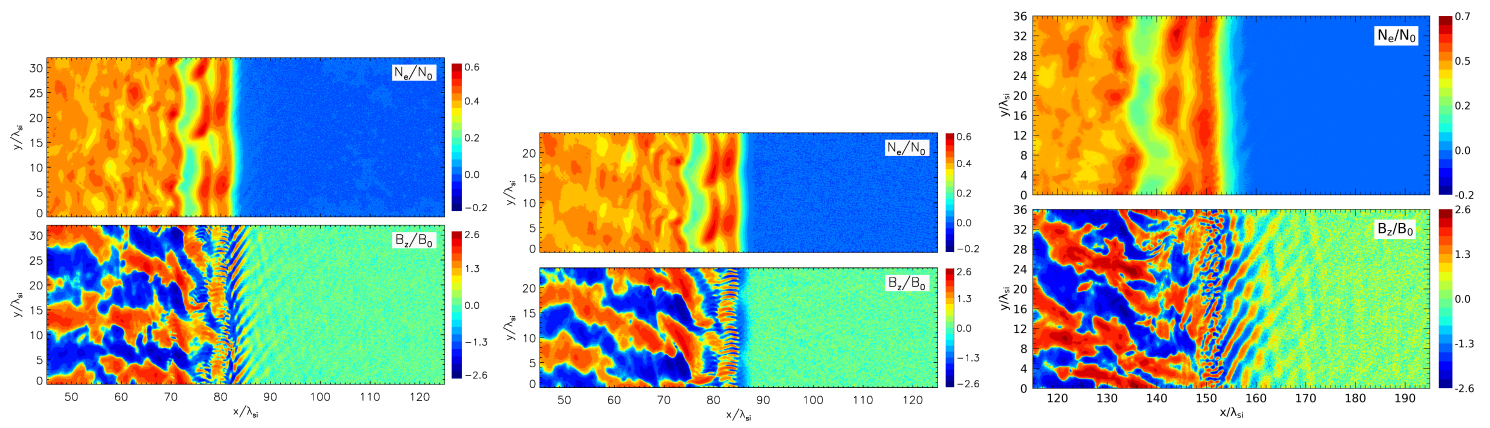

Figure 1: Shock structure at the rippled stage at time $\Omega_{\mathrm{i}} t=36$ for run A (left), run B (middle) and run $\mathrm{D}$ (right). Shown are distributions of the normalized electron number density, $N_{\mathrm{e}} / N_{0}$ (top panels), and the normalized magnetic field, $B_{z} / B_{0}$ (bottom panels). The density maps have logarithmic scaling. The scaling for magnetic fields is also logarithmic, but sign-preserving, and, e.g., for $B_{z}$ it is: $\operatorname{sgn}\left(B_{z}\right) \cdot\{2+$ $\left.\log \left[\max \left(\left|B_{z}\right| / B_{0}, 10^{-2}\right)\right]\right\}$. The level of "0" in the color scale hence corresponds to $\left|B_{z}\right| / B_{0} \leq 10^{-2}$.

The Alfvén Ion Cyclotron (AIC) instability operates at the shock front and near downstream. It is triggered by shock-reflected ions that give rise to the ion temperature anisotropy, $T_{\mathrm{i} \perp}>T_{\mathrm{i} \|}$, where $T_{\mathrm{i} \|}$ and $T_{\mathrm{i} \perp}$ are, respectively, the temperature components parallel and perpendicular to the local magnetic field. The AIC instability is responsible for the formation of shock rippling. The ripple modes have the longest wavelengths at the shock surface. Rippling in the second overshoot emerge earlier, their wavelengths are smaller, but grow with time. The electromagnetic structure further downstream suggests that also the mirror instabilities may operate there, generating longwavelength modes. The overshoot-undershoot system also hosts short-scale waves, visible in maps of $B_{z}$ magnetic field component (bottom panels in Fig. 1). These modes are identified with Whistler waves driven by the electron temperature anisotropy, $T_{e, \perp}>T_{e, \|}$. The growth of $T_{e, \perp}$ results from adiabatic compression of electrons gyrating fast in the overshoots.

The ripples and Whistler waves have similar structures in runs $\mathrm{A}$ and $\mathrm{B}$, but some differences occur with increasing plasma beta. In runs $\mathrm{A}$ and $\mathrm{B}$ with $\beta=5$ the ripples at the shock front have wavelength $\lambda_{\text {rippl }} \approx 16 \lambda_{\text {si }}$, which is agreement with $\lambda_{\mathrm{AIC}} \approx 16.5 \lambda_{\mathrm{si}}$ from the linear dispersion analysis [9]. The ripple wave in run D has a larger wavelength, $\lambda_{\text {rippl }} \approx 20 \lambda_{\text {si }}$, in line with linear theory and numerical simulations which show an increasing wavelength of the AIC-driven modes with growing plasma beta [8]. Longer wavelengths are observed also in the rippled structure of the second overshoot. The Whistlers are generated in the laminar stage. Their wavelength is independent of plasma beta, but they are stronger for higher $\beta$, consistent with theoretical expectations [5]. For $\beta \gtrsim 10$, the Whistler waves occur predominantly in the first overshoot. In the second overshoot they are damped via electron scattering. In the turbulent shock stage the modes are also distorted by EFI waves inflowing with the upstream plasma (see the right bottom panel in Fig. 1).

Electron reflection in the SDA process provides electrons streaming along the upstream magnetic field lines. The resulting electron temperature anisotropy, $T_{\mathrm{e} \|}>T_{\mathrm{e} \perp}$, excites the oblique modes of the EFI. As noted, due to conditions of $v_{\text {th, }} \lesssim v_{\mathrm{t}}$ assumed in our simulations, EFI waves are weakly driven in the laminar shock stage in runs $\mathrm{A}, \mathrm{C} 1-\mathrm{C} 4$, and $\mathrm{D}$ with $\theta_{\mathrm{Bn}}=75^{\circ}$. However, they are significantly amplified in rippled shocks, in which combined effects of a smaller crossshock potential jump and variable magnetic field compression along the shock front result in the 



Figure 2: Color-coded evolution of the downstream (left) and upstream (right) electron spectra for the simulation runs discussed in this work: $\beta=5, \theta_{\mathrm{Bn}}=75^{\circ}$ (top, run $\mathrm{A}$ ), $\beta=5, \theta_{\mathrm{Bn}}=78^{\circ}$ (middle, run $\mathrm{B}$ ), and $\beta=20, \theta_{\mathrm{Bn}}=75^{\circ}$ (bottom, run D). Maxwellian fits to the low-energy parts of the spectra are shown with dotted lines. Straight dotted lines at high momenta in downstream spectra denote a power-law of slope $p$. Magenta curves in upstream spectra display predictions of the SDA theory.

increased electron reflection. The EFI mode structure is formed by two dominant wave vectors, whose inclination is symmetric with respect to the upstream magnetic field direction at an angle $\theta_{\mathrm{Bk}} \approx 67^{\circ}[9$, see also $[3,4,6]]$. This structure is evident in Figure 1 (left and right panels). In high- $\beta$ runs $\mathrm{C} 2-\mathrm{C} 4$ and $\mathrm{D}$, the EFI modes are also stronger and have larger wavelength than in run A. On the other hand, in run B with $\theta_{\mathrm{Bn}}=78^{\circ}$, the EFI waves are not generated (see Section 2). 

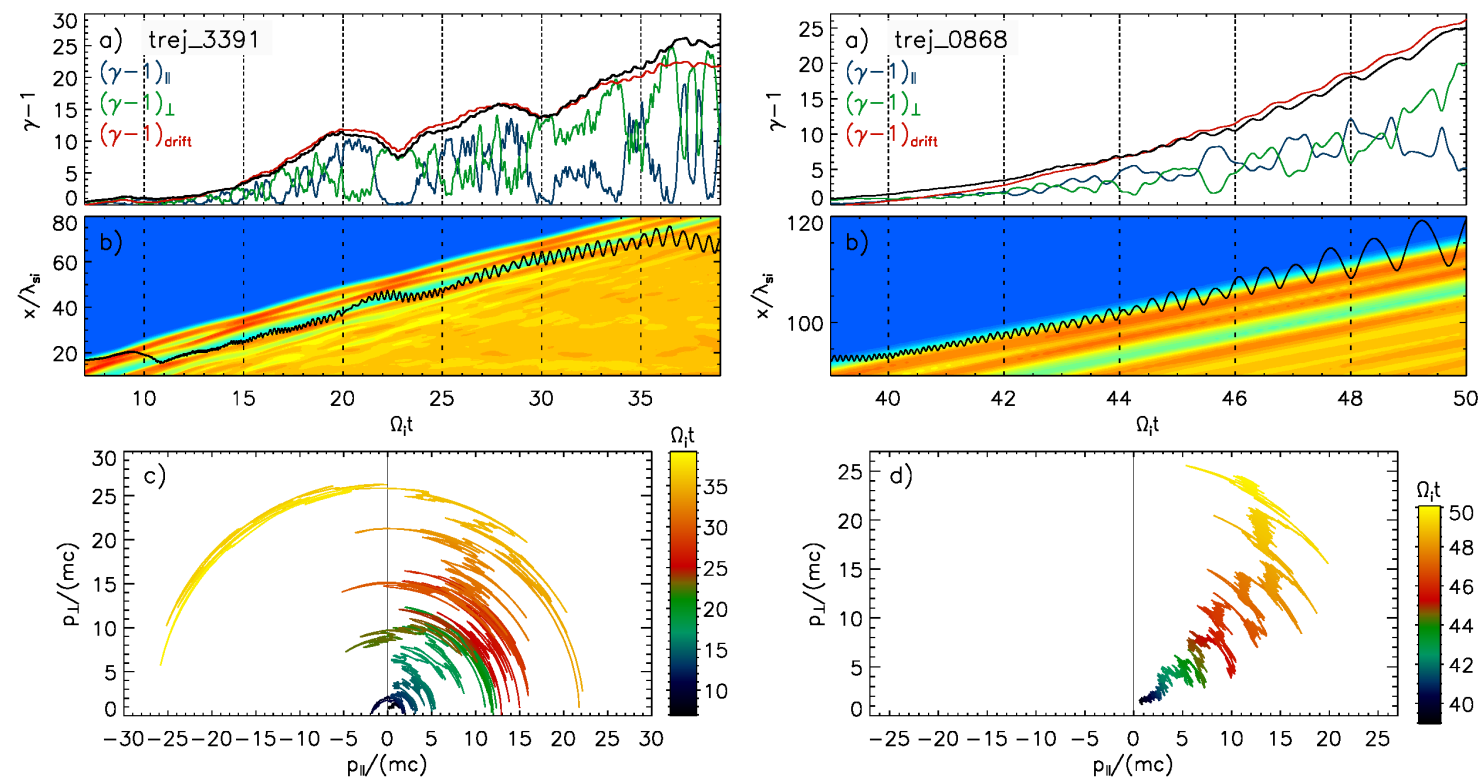

Figure 3: Trajectories of typical particles accelerated in run A (left) and run B (right). Shown are: time evolution of energy (black line) and that expected for drift along the motional $E_{z}$-field (red line; top panels), the particle location relative to the shock in the $x$-direction overlaid on the $y$-averaged density profile (middle panels), and particle orbits in $p_{\|}-p_{\perp}$ momentum space with color-coded time-scale (bottom panels). All quantities are measured in the downstream rest frame.

Figure 2 shows the time evolution of the electron energy spectra $20-28 \lambda_{s i}$ downstream (left) and $6-14 \lambda_{s i}$ upstream (right) of the shock. The spectra are compared up to $t_{\text {max }} \Omega_{\mathrm{i}}=50$ (compare Table 1). Magenta lines in the upstream spectra show a synthetic spectrum of electrons accelerated in a single SDA cycle [3]. One can note that early time spectra for run A with $\beta=5$ and $\theta_{\mathrm{Bn}}=75^{\circ}$ (and up to $\beta \approx 10$; runs $\mathrm{C} 1-\mathrm{C} 2$ ) largely follow the theoretical spectrum, though deviations from this prediction can be observed already in the laminar stage. This demonstrates that at this phase other processes than SDA contribute to the electron energization. The acceleration rate significantly increases after the appearance of shock rippling at $\Omega_{\mathrm{i}} t \approx 25$. Final non-thermal spectral tails have a SDA-like shape, but extend to much higher energy than the standard SDA theory predicts. The Lorentz factor needed for injection to DSA can be estimated as as a few $p_{\text {th,i }} / m_{e} c$ [6], which gives $\gamma_{\text {inj }} \approx 25$. In run A the electrons reach $\gamma_{\max } \approx 40$ at time $t \Omega_{\mathrm{i}}=50$ (and $\gamma_{\max } \approx 60$ at $t \Omega_{\mathrm{i}}=78$ [9]), so that the injection condition to DSA is well satisfied.

Electrons are accelerated mostly via SSDA mechanism which is based on the electron confinement at the shock through pitch-angle scattering off multi-scale turbulence in the shock transition. Most particles gain energies at a single interaction with the shock and the acceleration time is considerably longer than a few $\Omega_{\mathrm{i}}^{-1}$, as in pure SDA [10]. Multiple-cycle SDA process is rare due to inefficient scattering off upstream waves. The highest-energy electrons are generated in interactions with long-wave ripple modes in the shock front. However, majority of the particles that populate the high-energy spectral tails gain their energy behind the shock. A particle shown in Figure 3 (left) is an example of that. The energy gain is achieved mostly through the drift along the motional electric field, $\Delta \gamma_{\text {drift }}=\left(-e / m_{\mathrm{e}} c^{2}\right) \int E_{z} d z$, as in SDA (compare red and black lines in top panel in Fig. 3). 
The trajectories in $p_{\|}-p_{\perp}$ space show that most of the acceleration is associated with an increase of the perpendicular momentum, in between which rapid pitch-angle scattering occurs, visible in $p_{\|}-p_{\perp}$ plots as arcs at constant total momentum (bottom panel in Fig. 3).

Efficient electron energization to $\gamma_{\max } \approx 40$ is also observed in run B. Due to the absence of upstream turbulence supra-thermal spectral tails are generated solely through SSDA. It introduces significant departures from theoretical SDA spectra already in the laminar shock stage. The formation of supra-thermal tails is gradual, unlike in run A, in which low-density population of particles with high Lorentz factor develops first, after the AIC shock surface modes appear. This is because electrons are mostly accelerated in interactions with the shock front, as the particle shown in Figure 3 (right). Electrons accelerated behind the shock do not contribute to upstream spectra because the configuration of the magnetic field which is close to the critical superluminality angle prohibits their escape upstream.

Upstream spectra obtained for runs $\mathrm{C} 2-\mathrm{C} 4$ and $\mathrm{D}$ with higher $\beta$ also show supra-thermal tails extending much beyond the single-SDA cycle predictions up to $\gamma_{\max } \approx 30$. When the rippling appears the energization rate increases significantly for all cases except run $\mathrm{C} 4$, for which $\gamma_{\max }$ shows a constant weak growth. This is probably due to fact that the electron energy spectrum significantly diverges from the single-SDA one already in the laminar shock phase, which is also observed in run D.

Due to the assumption of $v_{\text {th,e }} \approx v_{\mathrm{t}}$ in [5] the EFI modes are well developed in the laminar shock stage and become only slightly amplified by the rippling. Consequently, [5] note a significant contribution of multiple-cycle SDA to the spectrum formation, though acceleration of the highestenergy electrons proceeds via SSDA. Although our simulations for $\beta=5-10$ show that multiplecycle SDA is not critical for the electron injection, this process can effectively work at high plasma beta and $v_{\text {th,e }} \lesssim v_{\mathrm{t}}$ on account of stronger EFI waves generated in high- $\beta$ plasmas. Our results thus show that contributions of different electron acceleration mechanisms to the spectrum formation may vary depending on $\beta$.

Supra-thermal spectral tails are also formed downstream of the shock (left panels in Fig. 2). They have power-law dependence. In runs $\mathrm{A}$ and $\mathrm{C} 1$ these tails extend to $\gamma_{\max } \lesssim 20$ and have spectral index $p \approx 2.5$, in agreement with observed radio synchrotron spectra, $\alpha=(p-1) / 2 \approx 0.75$ [e.g., 14], though the latter require much larger Lorentz factors, $\gamma \gg \gamma_{\max }$. The downstream spectrum is mainly composed of electrons transmitted from upstream and accelerated around the second overshoot via SSDA with electron scattering off Whistlers and small- and medium-scale ripples. (compare Fig. 3 left). However, downstream spectra are steeper in run $\mathrm{B}$ with $\theta_{\mathrm{Bn}}=78^{\circ}$ and with increasing plasma beta. The power-law index changes from $p \approx 4.0$ in run $\mathrm{C} 2$ for $\beta=10$ to $p \approx 5.1$ in run $\mathrm{C} 4$ for $\beta=30$. We note that at higher plasma beta the strength of the ripple modes in the second overshoot region decreases and they are somewhat larger-wavelength than the modes observed for run A. Short-scale Whistlers are absent as well. Therefore, electron scattering necessary for SSDA is less effective than in conditions with $\beta=5$.

\section{Summary}

We have recently demonstrated the importance of the multi-scale turbulence including ion-scale shock rippling modes in effective electron injection at low Mach number ICM shocks in plasmas 
with $\beta=5$ and in conditions in which SDA electron reflection from the shock is moderately suppressed [9]. We have shown that the main pre-acceleration mechanism is stochastic SDA. In this work we extend our studies to cover a larger magnetic field obliquity angle and a range of plasma beta $\beta=5-30$. We show that SSDA operates in these conditions and the maximum Lorentz factors satisfy the condition for electron injection to DSA. Efficient acceleration via SSDA has been recently confirmed for high plasma beta by [5]. The upstream spectra were shown to weakly depend on $\beta$, which is also confirmed here. Our results thus imply that quasi-perpendicular ICM shocks that develop multi-scale turbulence should be able to inject thermal electrons to the DSA mechanism. Therefore, merger shocks in galaxy clusters remain plausible candidates for the sources of UHECRs.

\section{Acknowledgments}

This work has been supported by Narodowe Centrum Nauki through research projects 2019/33/B/ ST9/02569 (J.N.) and UMO-2016/22/E/ST9/00061 (O.K.). This research was supported by PLGrid Infrastructure. Numerical experiments were conducted on the Prometheus system at ACC Cyfronet AGH and also on resources provided by North German Supercomputing Alliance (HLRN) under projects bbp00014, and bbp00033.

\section{References}

[1] Brunetti, G., \& Jones, T. W. 2014, Int. J. Mod. Phys. D, 23, 1430007-98

[2] Buneman, O., Computer Space Plasma Physics: Simulation Techniques and Software Eds.: H. Matsumoto \& Y. Omura, Tokyo: Terra Scientific, 1993

[3] Guo, X., Sironi, L., \& Narayan, R. 2014, ApJ, 794, 153

[4] Guo, X., Sironi, L., \& Narayan, R. 2014, ApJ, 797, 47

[5] Ha, J.-H., Kim, S., Ryu, D., et al. 2021, arXiv:2102.03042

[6] Kang, H., Ryu, D., \& Ha, J.-H. 2019, ApJ, 876, 79

[7] Katou, T., \& Amano, T. 2019, ApJ, 874, 119

[8] Kim, S., Ha, J.-H., Ryu, D., et al. 2020, ApJ, 892, 85

[9] Kobzar, O., Niemiec, J., Amano, T., et al. 2021, ApJ accepted, arXiv:2107.00508

[10] Krauss-Varban, D., \& Wu, C., S. 1989, JGR, 94, 15367

[11] Matsukiyo, S., Ohira, Y.,Yamazaki, R., \& Umeda, T. 2011, ApJ, 742, 47

[12] Matsukiyo, S., \& Matsumoto, Y. 2015, Journal of Physics Conference Series, 642, 012017

[13] Niemiec, J., Pohl, M., Stroman, T., et al. 2008, ApJ, 684, 1174.

[14] van Weeren, R. J., Röttgering, H. J. A., Brüuggen, M., \& Hoeft, M. 2010, Science, 330, 347 